04

\title{
Рассеяние света малыми многослойными частицами: обобщенный метод разделения переменных
}

\author{
(C) В.Г. Фарафонов, В.И. Устимов
}

Государственный университет аэрокосмического приборостроения, 190000 Санкт-Петербург, Россия

e-mail: far@aanet.ru

Поступила в редакцию 25.08.2017 г.

Построено приближение Релея для рассеяния света малыми многослойными осесимметричными частицами, в котором их поляризуемость определяется с помощью обобщенного метода разделения переменных (SVM). В рамках этого метода скалярные потенциалы, градиент которых дает напряженность электрического поля, представляются в виде разложений по сферическим гармоникам уравнения Лапласа. Неизвестные коэффициенты разложений определяются из граничных условий, которые сводятся к бесконечным системам линейных алгебраических уравнений (БСЛАУ), поскольку нет полного разделения переменных. Определена $T$-матрица электростатической задачи, главный элемент которой $T_{11}$ пропорционален поляризуемости частицы. Необходимое условие разрешимости БСЛАУ для метода SVM совпадает с условием корректного применения метода расширенных граничных условий (ЕВCM). Однако численные расчеты, при которых решаются конечномерные (т.е. редуцированные) системы, дают разные результаты в областях изменения параметров, близких к границе области применимости. Анализ численных расчетов сечений рассеяния и поглощения для двухслойных софокусных сфероидов, для которых имеется точное решение с использованием сфероидальных гармоник, показал преимущество метода SVM в сравнении с методом ЕВСМ. Оказалось, что предлагаемый метод дает пригодные результаты в более широкой области изменения параметров. Даже вне области применимости, где его следует рассматривать как некоторое приближенное решение, его использование в ряде случаев вполне допустимо. Дополнительные расчеты для трехслойных несофокусных сфероидов, а также трехслойных подобных псевдосфероидов и улиток Паскаля, которые можно получить из сфероидов в результате инверсии относительно начала координат и одного из фокусов соответственно, подтвердили данные выводы. Отметим, что последние частицы при определенных значениях параметров имеют невыпуклую форму.

DOI: $10.21883 /$ OS.2018.02.45533.212-17

\section{Введение}

Рассеяние света малыми двухслойными частицами в приближении Релея для софокусных эллипсов и сфероидов подробно рассматривается в монографиях [1-3]. Этот подход на случай многослойных конфокальных эллипсоидов обобщен в работе [4]. Дальнейшее обобщение для несофокусных эллипсоидов [5,6] нельзя признать удачным, поскольку оно работает в очень узкой области. Недавно [7,8] была предложена схема построения релеевского приближения для многослойных частиц с использованием метода расширенных граничных условий (ЕВСМ) для решения соответствующей электростатической задачи. Однако область его применимости распространяется на частицы не слишком сильно отклоняющиеся по форме от конфокальных сфероидов. В то же время известно [9], что для однородных осесимметричных частиц обобщенный метод разделения переменных (SVM) в рамках приближения Релея дает лучшие результаты по сравнению с ЕВСМ. Данное утверждение подтверждается сравнением этих двух методов в волновом случае как для однородных $[10,11]$, так и для слоистых частиц $[12,13]$.

В настоящей работе рассматривается приближение Релея для многослойных осесимметричных частиц с ис- пользованием SVM-решения соответствующей электростатической задачи. Обсуждается область применимости сферического базиса при использовании методов SVM и EBCM. В заключительной части работы анализируются результаты численных расчетов сечений рассеяния и поглощения для двух- и трехслойных конфокальных и неконфокальных сфероидов, а также подобных псевдосфероидов и улиток Паскаля, полученных с помощью SVM и ЕВCM.

Сечение поглощения и сечение рассеяния в приближении Релея определяются поляризуемостью частицы [1], а именно

$$
\begin{gathered}
C^{\mathrm{abs}}=k \operatorname{Im}\left(l^{2} \alpha_{x}+m^{2} \alpha_{y}+n^{2} \alpha_{z}\right), \\
C^{\mathrm{sca}}=\frac{k^{4}}{6 \pi}|\alpha|^{2}=\frac{k^{4}}{6 \pi}\left(l^{2}\left|\alpha_{x}\right|^{2}+m^{2}\left|\alpha_{y}\right|^{2}+n^{2}\left|\alpha_{z}\right|^{2}\right),
\end{gathered}
$$

где $k$ - волновое число, $l, m, n-$ направляющие косинусы напряженности внешнего электрического поля $\mathbf{E}_{0}$ относительно трех главных осей тензора поляризуемости. В приведенных выше формулах тензор поляризуемости предполагается диагональным, что справедливо для осесимметричных частиц. Для определения поляризуемости частицы следует решить соответствующую электростатическую задачу. 


\section{Постановка и SVM-решение электростатической задачи}

Рассмотрим многослойную осесимметричную частицу в постоянном электрическом поле. В сферической системе координат $(r, \theta, \varphi)$ уравнения поверхностей $S_{j}$ ее слоев можно записать в виде

$$
r_{j}=r_{j}(\theta)
$$

где $j=1,2, \ldots, J$. Будем считать, что ось $z$ декартовой системы $(x, y, z)$ совпадает с осью симметрии частицы. Все поверхности должны быть звездными, т.е. радиус-вектор, проведенный в любую точку, не должен пересекать поверхность дважды, при этом значение индекса $j=1$ соответствует внешней границе частицы, а $j=J-$ границе ее ядра.

При рассмотрении электростатических задач целесообразно использовать скалярные потенциалы $\Phi$, связанные с напряженностью электрического поля $\mathbf{E}=-\nabla \Phi$. Из уравнений Максвелла следует, что эти потенциалы удовлетворяют уравнению Лапласа $[1,3]$. Потенциал поля в $j$-й оболочке представим в виде суммы двух слагаемых $\Phi_{1}^{j+1}+\Phi_{2}^{j+1}$, причем первое слагаемое регулярно (т.е. конечно) в начале координат, а второе иррегулярно, т. е. имеет в начале координат особую точку. На бесконечности первое слагаемое постоянно или неограниченно растет, а второе - стремится к нулю. Внешнее поле вне частицы постоянно и описывается потенциалом $\Phi_{1}^{1}$, а поле во внешности частицы, возникающее из-за ее наличия, описывается потенциалом $\Phi_{2}^{1}$. Следует отметить, что в силу физических соображений иррегулярная часть внутреннего поля в ядре должна отсутствовать, т.е. $\Phi_{2}^{J+1}=0$ и остается только регулярный потенциал $\Phi_{1}^{J+1}$. Выше потенциалы с нижним индексом 1 регулярны (т. е. конечны) в начале координат, а потенциалы с нижним индексом 2 убывают к нулю на бесконечности, при этом они имеют особенность в начале координат. Верхний индекс потенциала указывает на номер слоя, в котором он описывает поле.

На поверхности раздела сред должны быть непрерывны тангенциальные составляющие напряженностей электрических полей и нормальные составляющие векторов электрической индукции $\mathbf{D}=\varepsilon \mathbf{E}[1,3]$, поэтому граничные условия можно записать в виде

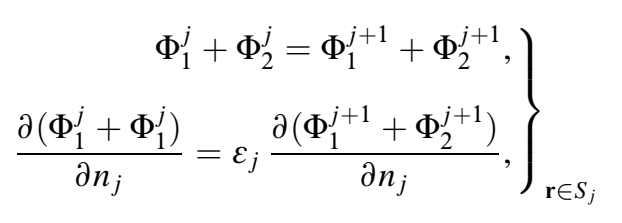

где $j=1,2, \ldots, J$, при этом $\frac{\partial}{\partial n_{j}}-$ производная вдоль внешней нормали к поверхности $S_{j}$. Кроме того, $\varepsilon_{j+1}-$ относительная диэлектрическая проницаемость для среды в $(j+1)$-й оболочке по сравнению со средой в $j$-й оболочке, т.е. $\varepsilon_{j}=\tilde{\varepsilon}_{j+1} / \tilde{\varepsilon}_{j}$, где $\tilde{\varepsilon}_{j}-$ комплексная диэлектрическая проницаемость среды в $j$-й оболочке.
Скалярные потенциалы, удовлетворяющие уравнению Лапласа, могут быть представлены в виде разложений по его сферическим гармоникам:

$$
\begin{aligned}
& \begin{array}{l}
\Phi_{1}^{j} \\
\Phi_{2}^{j}
\end{array}=\sum_{m=0}^{\infty} \sum_{l=m}^{\infty} \begin{array}{l}
a_{m l}^{j} r^{l} \\
b_{m l}^{j} \frac{1}{2 l+1} r^{-(l+1)}
\end{array} \psi_{m l}(\theta, \varphi) \\
& =\sum_{m=0}^{\infty} \sum_{l=m}^{\infty} \begin{array}{ll}
a_{m l}^{j} & \Psi_{m l}^{(1)}(\mathbf{r}) \\
b_{m l}^{j} & \Psi_{m l}^{(3)}(\mathbf{r}) .
\end{array}
\end{aligned}
$$

Заметим, что подобная запись справедлива также и для потенциалов внешнего и „рассеянного“ полей при $j=1$. Важно отметить, что радиальные функции $r^{l}$ входят в функции $\Psi_{m l}^{(1)}$ и обеспечивают конечность потенциалов $\Phi_{1}^{j}$ регулярных полей в начале координат, а радиальные функции $r^{-(l+1)}$ входят в функции $\Psi_{m l}^{(3)}$ и обеспечивают убывание к нулю потенциалов $\Phi_{2}^{j}$ иррегулярных полей на бесконечности. Угловые функции

$$
\psi_{m l}(\theta, \varphi)=\begin{gathered}
\psi_{m l e}(\theta, \varphi) \\
\psi_{m l o}(\theta, \varphi)
\end{gathered}=\bar{P}_{l}^{m}(\cos \theta) \sqrt{\frac{2-\delta_{m}^{0}}{2 \pi}} \cos m \varphi,
$$

образуют полную ортонормированную систему в пространстве $L^{2}(\Omega)$ с квадратичной метрикой на любой сфере $\Omega$ с центром в начале координат [2]. Здесь

$$
\bar{P}_{l}^{m}(\cos \theta)=\sqrt{\frac{(2 l+1)}{2} \frac{(l-m) !}{(l+m) !}} P_{l}^{m}(\cos \theta)
$$

- нормированные присоединенные функции Лежандра 1-го рода, символ Кронекера $\delta_{m}^{0}=1$ или 0 , когда $m=0$ и $m \neq 0$ соответственно.

Предположим, что единичное внешнее поле направлено вдоль оси $z$, т. е. $\mathbf{E}_{1}^{1}=\mathbf{i}_{z}$. Соответствующий скалярный потенциал имеет вид

$$
\Phi_{1}^{1}=-z=-r \cos \theta=a_{01}^{1} \Psi_{01}^{(1)}(\mathbf{r}),
$$

при этом отличен от нуля только коэффициент $a_{01}^{1}=-\sqrt{4 \pi / 3}$. Аналогично, если единичное внешнее поле направлено вдоль оси $x$ или оси $y$, то отличен от нуля только коэффициент $a_{01 e}^{1}=-\sqrt{4 \pi / 3}$ или $a_{01 o}^{1}=-\sqrt{4 \pi / 3}$.

Отметим, что в силу коммутативности оператора $T$ [14], соответствующего электростатической задаче для осесимметричных частиц, и оператора $L_{\mathrm{z}}=\frac{\partial}{\partial \varphi}$ азимутальный индекс $m=0$, если внешнее поле направлено вдоль оси $z$ (7). При ориентации внешнего поля перпендикулярно оси $z$ азимутальный индекс $m=1$.

Введем векторы $\mathbf{a}^{j}=\left\{a_{m l}^{j}\right\}_{m}^{\infty}, \mathbf{b}^{j}=\left\{b_{m l}^{j}\right\}_{m}^{\infty}-$ коэффициентов разложений потенциалов $\Phi_{1}^{j}$ и $\Phi_{2}^{j}$, т.е. потенциалов регулярных и иррегулярных полей соответственно. Кроме того, обозначим через $\tilde{\Psi}_{m l}$ сферические гармоники без множителей, зависящих от азимутального угла. 
Неизвестные коэффициенты разложений определяются из граничных условий. Подставим разложения (5) в граничные условия (4). Первые уравнения умножим на угловые функции $\left(\psi_{m n} \sin \theta\right)$, а вторые уравнения на $\left(\psi_{m n} r \sqrt{r^{2}+r_{\theta}^{\prime 2}} \sin \theta\right)$. Затем полученные выражения проинтегрируем по $\theta$ и $\varphi$ от нуля до $\pi$ и $2 \pi$ соответственно. В результате, учитывая разделение относительно азимутального угла, для определения неизвестных коэффициентов получается БСЛАУ, которая в матричном виде записывается следующим образом:

$$
\left(\begin{array}{ll}
\alpha_{11}^{j} & \alpha_{12}^{j} \\
\alpha_{21}^{j} & \alpha_{22}^{j}
\end{array}\right)\left(\begin{array}{l}
\mathbf{b}^{j} \\
\mathbf{a}^{j}
\end{array}\right)=\left(\begin{array}{cc}
\alpha_{11}^{j} & \alpha_{12}^{j} \\
\varepsilon_{j} \alpha_{21}^{j} & \varepsilon_{j} \alpha_{22}^{j}
\end{array}\right)\left(\begin{array}{l}
\mathbf{b}^{j+1} \\
\mathbf{a}^{j+1}
\end{array}\right),
$$

где матричные элементы выражаются через интегралы

$$
\begin{aligned}
\left(\alpha_{11}^{j}\right)_{n l}= & \int_{0}^{\pi} \tilde{\Psi}_{m l}^{(3)}(\theta) \bar{P}_{n}^{m}(\cos \theta) \sin \theta \mathrm{d} \theta \\
= & \int_{0}^{\pi} \bar{P}_{n}^{m}(\cos \theta) \frac{r^{-(l+1)}(\theta)}{2 l+1} \bar{P}_{l}^{m}(\cos \theta) \sin \theta \mathrm{d} \theta \\
\left(\alpha_{12}^{j}\right)_{n l}= & \int_{0}^{\pi} \tilde{\Psi}_{m l}^{(1)}(\theta) \bar{P}_{n}^{m}(\cos \theta) \sin \theta \mathrm{d} \theta \\
& =\int_{0}^{\pi} \bar{P}_{n}^{m}(\cos \theta) r^{l}(\theta) \bar{P}_{l}^{m}(\cos \theta) \sin \theta \mathrm{d} \theta, \\
\left(\alpha_{21}^{j}\right)_{n l}= & \int_{0}^{\pi} \frac{\partial \tilde{\Psi}_{m l}^{(3)}(\theta)}{\partial n} \bar{P}_{n}^{m}(\cos \theta) r \sqrt{r^{2}+r_{\theta}^{\prime 2}} \sin \theta \mathrm{d} \theta \\
= & \int_{0}^{\pi} \bar{P}_{n}^{m}(\cos \theta) \frac{r^{-l}(\theta)}{2 l+1}\left(-(l+1) \bar{P}_{l}^{m}(\cos \theta)\right. \\
& \left.+\frac{r(\theta)^{\prime}}{r(\theta)} \sin \theta \bar{P}_{l}^{m \prime}(\cos \theta)\right) \sin \theta \mathrm{d} \theta, \\
= & \int_{0}^{\pi} \bar{P}_{n}^{m}(\cos \theta) r^{l+1}(\theta)\left(l \bar{P}_{l}^{m}(\cos \theta)\right. \\
\left(\alpha_{22}^{j}\right)_{n l}= & \int_{0}^{\pi} \frac{\partial \tilde{\Psi}_{m l}^{(1)}(\theta)}{\partial n} \bar{P}_{n}^{m}(\cos \theta) r \sqrt{r^{2}+r_{\theta}^{\prime 2}} \sin \theta \mathrm{d} \theta \\
& \left.\sin \theta \bar{P}_{l}^{m \prime}(\cos \theta)\right) \sin \theta \mathrm{d} \theta . \\
&
\end{aligned}
$$

Заметим, что эти интегралы зависят от индекса $j$ через функцию $r_{j}(\theta)$, описывающую поверхность $j$-го слоя частицы.
Введем обозначение для обратной матрицы:

$$
\left(\begin{array}{ll}
\alpha_{11}^{j} & \alpha_{12}^{j} \\
\alpha_{21}^{j} & \alpha_{22}^{j}
\end{array}\right)^{-1}=\left(\begin{array}{ll}
f_{11}^{j} & f_{12}^{j} \\
f_{21}^{j} & f_{22}^{j}
\end{array}\right)
$$

Теперь БСЛАУ можно записать в виде

$$
\left(\begin{array}{c}
\mathbf{b}^{j} \\
\mathbf{a}^{j}
\end{array}\right)=\left(I_{F}+\left(\varepsilon_{j}-1\right) F^{j}\right)\left(\begin{array}{c}
\mathbf{b}^{j+1} \\
\mathbf{a}^{j+1}
\end{array}\right),
$$

где

$$
\begin{gathered}
F^{j}=\left(\begin{array}{cc}
f_{11}^{j} & f_{12}^{j} \\
f_{21}^{j} & f_{22}^{j}
\end{array}\right)\left(\begin{array}{cc}
0 & 0 \\
\alpha_{21}^{j} & \alpha_{22}^{j}
\end{array}\right)=\left(\begin{array}{lll}
f_{12}^{j} \alpha_{21}^{j} & f_{12}^{j} \alpha_{22}^{j} \\
f_{22}^{j} \alpha_{21}^{j} & f_{22}^{j} \alpha_{22}^{j}
\end{array}\right), \\
I_{F}=\left(\begin{array}{ll}
I & 0 \\
0 & I
\end{array}\right) .
\end{gathered}
$$

Решение БСЛАУ (11) можно представить в виде

$$
\begin{aligned}
\left(\begin{array}{c}
\mathbf{b}^{\mathbf{1}} \\
\mathbf{a}^{\mathbf{1}}
\end{array}\right)= & \left(I_{F}+\left(\varepsilon_{1}-1\right) F^{1}\right)\left(I_{F}+\left(\varepsilon_{2}-1\right) F^{2}\right) \ldots \\
& \left(I_{F}+\left(\varepsilon_{J}-1\right) F^{J}\right)\left(\begin{array}{c}
\mathbf{0} \\
\mathbf{a}^{J+1}
\end{array}\right) .
\end{aligned}
$$

Окончательное решение электростатической задачи описывается $T$-матрицей, которая связывает коэффициенты разложений „рассеянного“ и внешнего полей:

$$
\mathbf{b}^{\mathbf{1}}=T \mathbf{a}^{\mathbf{1}} .
$$

Принимая во внимание соотношения (13), для $T$-матрицы получим

$$
T=A_{1} A_{2}^{-1},
$$

где

$$
\begin{aligned}
\left(\begin{array}{l}
A_{1} \\
A_{2}
\end{array}\right)= & \left(I_{F}+\left(\varepsilon_{1}-1\right) F^{1}\right) \ldots\left(I_{F}+\left(\varepsilon_{J-1}-1\right) F^{J-1}\right) \\
& \times\left(\begin{array}{c}
\left(\varepsilon_{J}-1\right) f_{12}^{J} \alpha_{22}^{J} \\
I+\left(\varepsilon_{J}-1\right) f_{22}^{J}
\end{array}\right),
\end{aligned}
$$

матрицы $I_{F}$ и $F^{j}$ являются блочными (12).

Для $T$-матрицы наиболее важным является элемент $T_{11}$, который связывает единственный отличный от нуля коэффициент $a_{01}^{1}$ внешнего поля (7) с коэффициентом $b_{01}^{1}$. В дальней зоне данный коэффициент соответствует дипольному слагаемому, которое дает основной вклад в „рассеянное“ поле. Важным свойством $T$-матрицы является ее симметричность [15].

В релеевском приближении для вычисления сечений рассеяния и поглощения, а также других характеристик рассеянного излучения используется тензор поляризуемости частицы (1), (2). Для осесимметричных частиц (3) данный тензор является диагональным и может быть определен с помощью $T$-матрицы. В рассматриваемом 
случае при ориентации внешнего поля вдоль оси вращения частицы имеем [16]

$$
\alpha_{z}=\frac{4 \pi}{3} \frac{b_{01}^{1}}{a_{01}^{1}}=\frac{4 \pi}{3} T_{01}^{01},
$$

при этом в знаменателе находится коэффициент разложения потенциала внешнего поля, а в числителе - коэффициент разложения потенциала „рассеянного“ поля.

\section{Область применения SVM}

Ранее [7] была исследована область применимости ЕВСМ в электростатической задаче для многослойных осесимметричных частиц. Аналитический и численный анализ показал, что в общем случае, а именно, исключая многослойные сфероиды, БСЛАУ в рамках ЕВСМ имеют единственное решение, которое можно найти методом редукции с любой точностью при выполнении условия

$$
\max \left\{\sigma_{1}^{(j)}\right\}<\min \left\{\sigma_{2}^{(j)}\right\} .
$$

Здесь параметры $\sigma_{1}^{(j)}$ и $\sigma_{2}^{(j)}$ равны расстояниям до первых особых точек, встречающихся при аналитической деформации $j$-й поверхности частицы до сфер соответственно во внутренность и внешность (подробнее см. $[14,17])$. Данное условие можно связать с радиусами сходимости разложений регулярных и иррегулярных полей вне и внутри частицы, включая ее оболочки, так как $R_{1}^{(j)}=\sigma_{1}^{(j)}, R_{2}^{(j)}=\sigma_{2}^{(j)}$. Иначе говоря, для разрешимости БСЛАУ должна существовать сферическая оболочка, в которой одновременно сходятся разложения всех регулярных и иррегулярных полей. Это требование является естественным обобщением результата для однородных частиц, для которых подобное условие накладывается только на разложения „рассеянного“ и внутреннего полей $-R_{1}<R_{2}$.

Для сфероидальных многослойных частиц, которые следует выделить в отдельный класс, условие применимости ЕВСМ более слабое (отсутствует параметр $\sigma_{2}^{(j)}$ для ядра частицы) и записывается в виде

$$
\begin{array}{r}
\max \left\{\sigma_{1}^{(1)}, \sigma_{1}^{(2)}, \ldots, \sigma_{1}^{(J-1)}, \sigma_{1}^{(J)}\right\} \\
<\min \left\{\sigma_{2}^{(1)}, \sigma_{2}^{(2)}, \ldots, \sigma_{2}^{(J-1)}\right\},
\end{array}
$$

при этом параметры поверхностей оболочек $\sigma_{2}^{(j)}$ необязательно связаны с соответствующими радиусами сходимости $R_{2}^{j}$ иррегулярных полей. Например, условие (19) для двухслойного конфокального сфероида приводит к ограничению на отношение полуосей внешней оболочки:

$$
\frac{a_{1}}{b_{1}}<1+\sqrt{2} \approx 2.41
$$

в то время как условие $(18)$ дает $a_{1} / b_{1}<\sqrt{2} \approx 1.41$. В этом случае радиусы сходимости разложений „рассеянного“ поля и иррегулярной части поля внутри оболочки определяются фокусным расстоянием частицы

$$
R_{1}=\frac{d}{2}
$$

а поле внутри ядра и регулярная часть поля внутри оболочки постоянны, т. е. $R_{2}=\infty[18,11]$. В силу этого требование

$$
\max \left\{R_{1}^{(j)}\right\}<\min \left\{R_{2}^{(j)}\right\},
$$

т.е. требование наличия сферической оболочки, в которой одновременно сходились бы разложения всех регулярных и иррегулярных полей, привело бы к ошибочному выводу о применимости ЕВСМ для любых конфокальных сфероидов. В данном случае условия (19) и (22) не эквивалентны. Параметр

$$
\sigma_{2}^{(1)}=\frac{4 a_{1} b_{1}}{d_{1}}
$$

для внешней поверхности сфероида в неравенстве (19) реально присутствует, и в результате этого для применимости ЕВСМ в случае конфокальных сфероидов возникает условие (20). Численные расчеты с „произвольной“ точностью (т.е. с учетом 300 значащих цифр) подтверждают это утверждение с очевидной достоверностью [18]. Следует напомнить, что для однородных сфероидов ЕВСМ применимо всегда, при этом внутреннее поле постоянно и определяется в явном виде, а разложение „рассеянного“ поля имеет радиус сходимости в соответствии с формулой (21).

Для метода SVM приведенные выше утверждения для разрешимости БСЛАУ, возникающих в рамках ЕВСМ, должны оставаться справедливыми, так как решение задачи в обоих случаях ищется в виде одних и тех же разложений. С теоретической точки зрения, если решения БСЛАУ для двух методов существуют, то они идентичны, поэтому радиусы сходимости разложений полей одинаковы. При численных расчетах для определения неизвестных коэффициентов разложений решаются не БСЛАУ, а редуцированные, т. е. конечномерные системы линейных алгебраических уравнений. В этом случае результаты двух алгоритмов могут быть различными, что наблюдается в действительности. Во-первых, различаются матричные элементы и свободные члены систем для этих двух методов, и во-вторых, погрешности, неизбежно возникающие в процессе численных расчетов, также по-разному себя проявляют. Подробнее эти вопросы будут обсуждаться в следующем разделе.

\section{Результаты численных расчетов и их обсуждение}

Все численные расчеты, результаты которых обсуждаются ниже, проводились с удвоенной точностью, т.е. с 16 значащими цифрами, при этом алгоритмы вычислений интегральных матричных элементов были приведены в работах [7,9]. Время вычисления $T$-матрицы и характеристик рассеяния с использованием SVM 
Таблица 1. Результаты расчета сечений $(k=0.5)$ двухслойных конфокальных сфероидов в зависимости от числа уравнений $N$, учитываемых в БСЛАУ. Параметры внешней оболочки: $a_{1}=1, b_{1}=0.7, \tilde{\varepsilon}_{1}=1.69+0.10 j$; диэлектрическая проницаемость ядра $\tilde{\varepsilon}_{2}=2.25+0.15 j$. Здесь $\mathrm{ES}-$ точное решение задачи

\begin{tabular}{|c|c|c|c|c|c|c|c|c|}
\hline \multirow{2}{*}{$V_{2} / V_{1}$} & \multirow{2}{*}{$a_{2} / b_{2}$} & \multirow{2}{*}{$N$} & \multicolumn{3}{|c|}{ Сечение рассеяния } & \multicolumn{3}{|c|}{ Сечение поглощения } \\
\hline & & & ES & $\operatorname{EBCM}(N)$ & $\operatorname{SVM}(N)$ & ES & $\operatorname{EBCM}(N)$ & $\operatorname{SVM}(N)$ \\
\hline 0.9 & 1.461 & $\begin{array}{c}8 \\
16 \\
24 \\
32 \\
40 \\
\end{array}$ & $1.2175 \mathrm{E}-02$ & $\begin{array}{l}1.2167 \mathrm{E}-02 \\
1.2175 \mathrm{E}-02 \\
1.2175 \mathrm{E}-02 \\
1.2175 \mathrm{E}-02 \\
1.2175 \mathrm{E}-02 \\
\end{array}$ & $\begin{array}{l}1.2173 \mathrm{E}-02 \\
1.2175 \mathrm{E}-02 \\
1.2175 \mathrm{E}-02 \\
1.2175 \mathrm{E}-02 \\
1.2175 \mathrm{E}-02 \\
\end{array}$ & $1.1631 \mathrm{E}-01$ & $\begin{array}{l}1.1621 \mathrm{E}-01 \\
1.1631 \mathrm{E}-01 \\
1.1631 \mathrm{E}-01 \\
1.1631 \mathrm{E}-01 \\
1.1631 \mathrm{E}-01 \\
\end{array}$ & $\begin{array}{l}1.1630 \mathrm{E}-01 \\
1.1631 \mathrm{E}-01 \\
1.1631 \mathrm{E}-01 \\
1.1631 \mathrm{E}-01 \\
1.1631 \mathrm{E}-01 \\
\end{array}$ \\
\hline 0.7 & 1.547 & $\begin{array}{c}8 \\
16 \\
24 \\
32 \\
40\end{array}$ & $1.0366 \mathrm{E}-02$ & $\begin{array}{l}1.0360 \mathrm{E}-02 \\
1.0365 \mathrm{E}-02 \\
1.0366 \mathrm{E}-02 \\
1.0366 \mathrm{E}-02 \\
1.0366 \mathrm{E}-02\end{array}$ & $\begin{array}{l}1.0363 \mathrm{E}-02 \\
1.0366 \mathrm{E}-02 \\
1.0366 \mathrm{E}-02 \\
1.0366 \mathrm{E}-02 \\
1.0366 \mathrm{E}-02\end{array}$ & $1.0801 \mathrm{E}-01$ & $\begin{array}{l}1.0793 \mathrm{E}-01 \\
1.0800 \mathrm{E}-01 \\
1.0801 \mathrm{E}-01 \\
1.0801 \mathrm{E}-01 \\
1.0801 \mathrm{E}-01\end{array}$ & $\begin{array}{l}1.0799 \mathrm{E}-01 \\
1.0801 \mathrm{E}-01 \\
1.0801 \mathrm{E}-01 \\
1.0801 \mathrm{E}-01 \\
1.0801 \mathrm{E}-01 \\
\end{array}$ \\
\hline 0.5 & 1.687 & \begin{tabular}{c|}
8 \\
16 \\
24 \\
32 \\
40
\end{tabular} & $8.6760 \mathrm{E}-03$ & $\begin{array}{l}8.6724 \mathrm{E}-03 \\
8.6753 \mathrm{E}-03 \\
8.6759 \mathrm{E}-03 \\
8.6760 \mathrm{E}-03 \\
8.6760 \mathrm{E}-03\end{array}$ & $\begin{array}{l}8.6715 \mathrm{E}-03 \\
8.6760 \mathrm{E}-03 \\
8.6760 \mathrm{E}-03 \\
8.6760 \mathrm{E}-03 \\
8.6760 \mathrm{E}-03\end{array}$ & $9.9323 \mathrm{E}-02$ & $\begin{array}{l}9.9264 \mathrm{E}-02 \\
9.9314 \mathrm{E}-02 \\
9.9323 \mathrm{E}-02 \\
9.9323 \mathrm{E}-02 \\
9.9323 \mathrm{E}-02\end{array}$ & $\begin{array}{l}9.9295 \mathrm{E}-02 \\
9.9323 \mathrm{E}-02 \\
9.9323 \mathrm{E}-02 \\
9.9323 \mathrm{E}-02 \\
9.9323 \mathrm{E}-02\end{array}$ \\
\hline 0.3 & 1.969 & $\begin{array}{c}8 \\
16 \\
24 \\
32 \\
40 \\
\end{array}$ & $7.1086 \mathrm{E}-03$ & $\begin{array}{l}7.1068 \mathrm{E}-03 \\
7.1081 \mathrm{E}-03 \\
7.1084 \mathrm{E}-03 \\
7.1086 \mathrm{E}-03 \\
7.1086 \mathrm{E}-03 \\
\end{array}$ & $\begin{array}{l}7.0975 \mathrm{E}-03 \\
7.1085 \mathrm{E}-03 \\
7.1086 \mathrm{E}-03 \\
7.1086 \mathrm{E}-03 \\
7.1086 \mathrm{E}-03 \\
\end{array}$ & $9.0195 \mathrm{E}-02$ & $\begin{array}{l}9.0162 \mathrm{E}-02 \\
9.0187 \mathrm{E}-02 \\
9.0192 \mathrm{E}-02 \\
9.0195 \mathrm{E}-02 \\
9.0195 \mathrm{E}-02\end{array}$ & $\begin{array}{l}9.0119 \mathrm{E}-02 \\
9.0195 \mathrm{E}-02 \\
9.0195 \mathrm{E}-02 \\
9.0195 \mathrm{E}-02 \\
9.0195 \mathrm{E}-02 \\
\end{array}$ \\
\hline 0.1 & 2.982 & $\begin{array}{c}8 \\
16 \\
24 \\
32 \\
40\end{array}$ & $5.6611 \mathrm{E}-03$ & $\begin{array}{l}5.6606 \mathrm{E}-03 \\
5.6609 \mathrm{E}-03 \\
5.6610 \mathrm{E}-03 \\
5.6610 \mathrm{E}-03 \\
5.6611 \mathrm{E}-03\end{array}$ & $\begin{array}{l}5.6232 \mathrm{E}-03 \\
5.6587 \mathrm{E}-03 \\
6.3153 \mathrm{E}-03 \\
5.5648 \mathrm{E}-03 \\
5.7615 \mathrm{E}-03\end{array}$ & $8.0461 \mathrm{E}-02$ & $\begin{array}{l}8.0451 \mathrm{E}-02 \\
8.0459 \mathrm{E}-02 \\
8.0460 \mathrm{E}-02 \\
8.0460 \mathrm{E}-02 \\
8.0462 \mathrm{E}-02\end{array}$ & $\begin{array}{l}8.0165 \mathrm{E}-02 \\
8.0441 \mathrm{E}-02 \\
8.3971 \mathrm{E}-02 \\
7.9698 \mathrm{E}-02 \\
8.1245 \mathrm{E}-02\end{array}$ \\
\hline
\end{tabular}

примерно в 2 раза больше, чем при использовании ЕВСМ. Увеличение объема расчетов связано с тем, что в SVM-схеме (10)-(16) дополнительно требуется вычислять обратные матрицы $F^{j}(12)$, число которых равно количеству слоев частицы. Контроль за правильностью расчетов обеспечивается сравнением решений, полученных разными методами, включая явное точное решение для конфокальных сфероидов [19]. Кроме того, рассматривается сходимость результатов по мере увеличения количества учитываемых в разложениях слагаемых $N$. Наконец, проверяется условие симметричности $T$-матрицы, например, совпадение первой строки и первого столбца этой матрицы:

$$
T_{1 j}^{01}=T_{j 1}^{01}
$$

Данный тест быстро реагирует на уменьшение точности расчетов. Заметим, что в силу симметрии для сфероидов и псевдосфероидов $T_{i j}^{01}=0$ при $i-j=2 k+1$, т.е. половина матричных элементов $T$-матрицы равна нулю.

В табл. 1, 2 приведены значения сечений рассеяния и поглощения для двухслойных конфокальных сфероидов рассчитанные с помощью ЕBCM и SVM, область применимости которых определяется условием (20). Однако в этом случае имеется точное решение, которое можно записать в явном виде $[1,19]$. Если отношение полуосей частицы достаточно мало, например, $a_{1} / b_{1}=10 / 7 \approx 1.43$ и ядро не слишком вытянутое $a_{2} / b_{2}<2.5$, то влияние вычислительных погрешностей на результат расчета сечений в зависимости от количества учитываемых слагаемых $N$ в разложениях полей мало заметно (табл. 1). В этом случае размерность $N_{T}$ симметричного блока $T$ - матрицы, т.е. размерность усеченной $T$-матрицы, для которой свойство симметричности (24) выполняется с относительной погрешностью $10 \%$ (т.е. $\left.10^{-1}\right)$, достаточно велика, а именно $N_{T}>20$. При этом с увеличением $N$ точность расчетов увеличивается, т. е. наблюдается сходимость к точному решению. Этот результат справедлив как для EBCM, так и для SVM. Последний случай в этой таблице $a_{2} / b_{2}=2.982$ соответствует ситуации, при которой вычислительные погрешности сильно влияют на результаты расчетов. В рамках SVM-схемы наиболее точный результат (три значащих цифры) получается при $N=16$, далее с увеличением $N$ точность расчетов значительно уменьшается (только первая цифра для сечения поглощения). Этот случай является единственным, когда ЕВCM имеет преимущество против метода SVM. Здесь 
Таблица 2. То же, что в табл. 1 , но при $a_{1}=1$ и $b_{1}=0.5$

\begin{tabular}{|c|c|c|c|c|c|c|c|c|}
\hline \multirow{2}{*}{$V_{2} / V_{1}$} & \multirow{2}{*}{$a_{2} / b_{2}$} & \multirow{2}{*}{$N$} & \multicolumn{3}{|c|}{ Сечение рассеяния } & \multicolumn{3}{|c|}{ Сечение поглощения } \\
\hline & & & ES & $\operatorname{EBCM}(N)$ & $\operatorname{SVM}(N)$ & $\mathrm{ES}$ & $\operatorname{EBCM}(N)$ & $\operatorname{SVM}(N)$ \\
\hline 0.9 & 2.073 & $\begin{array}{c}8 \\
16 \\
24 \\
32 \\
40\end{array}$ & $3.6350 \mathrm{E}-03$ & $\begin{array}{l}3.3887 \mathrm{E}-03 \\
3.5694 \mathrm{E}-03 \\
3.6114 \mathrm{E}-03 \\
3.6166 \mathrm{E}-03 \\
3.6143 \mathrm{E}-03\end{array}$ & $\begin{array}{l}3.5870 \mathrm{E}-03 \\
3.6344 \mathrm{E}-03 \\
3.6350 \mathrm{E}-03 \\
3.6350 \mathrm{E}-03 \\
3.6341 \mathrm{E}-03\end{array}$ & $6.8052 \mathrm{E}-02$ & $\begin{array}{l}6.4864 \mathrm{E}-02 \\
6.7132 \mathrm{E}-02 \\
6.7745 \mathrm{E}-02 \\
6.7820 \mathrm{E}-02 \\
6.7865 \mathrm{E}-02\end{array}$ & $\begin{array}{l}6.7518 \mathrm{E}-02 \\
6.8045 \mathrm{E}-02 \\
6.8052 \mathrm{E}-02 \\
6.8052 \mathrm{E}-02 \\
6.7974 \mathrm{E}-02\end{array}$ \\
\hline 0.7 & 2.266 & $\begin{array}{c}8 \\
16 \\
24 \\
32 \\
40\end{array}$ & $3.0712 \mathrm{E}-03$ & $\begin{array}{l}2.8153 \mathrm{E}-03 \\
2.9917 \mathrm{E}-03 \\
3.0381 \mathrm{E}-03 \\
3.0418 \mathrm{E}-03 \\
3.0409 \mathrm{E}-03\end{array}$ & $\begin{array}{l}3.0197 \mathrm{E}-03 \\
3.0702 \mathrm{E}-03 \\
3.0712 \mathrm{E}-03 \\
3.0712 \mathrm{E}-03 \\
3.0769 \mathrm{E}-03\end{array}$ & $6.2699 \mathrm{E}-02$ & $\begin{array}{l}5.9095 \mathrm{E}-02 \\
6.1595 \mathrm{E}-02 \\
6.2205 \mathrm{E}-02 \\
6.2284 \mathrm{E}-02 \\
6.2306 \mathrm{E}-02\end{array}$ & $\begin{array}{l}6.2092 \mathrm{E}-02 \\
6.2687 \mathrm{E}-02 \\
6.2699 \mathrm{E}-02 \\
6.2699 \mathrm{E}-02 \\
6.2885 \mathrm{E}-02\end{array}$ \\
\hline 0.5 & 2.576 & $\begin{array}{c}8 \\
16 \\
24 \\
32 \\
40\end{array}$ & $2.5467 \mathrm{E}-03$ & $\begin{array}{l}2.2850 \mathrm{E}-03 \\
2.4398 \mathrm{E}-03 \\
2.4963 \mathrm{E}-03 \\
2.4922 \mathrm{E}-03 \\
2.4358 \mathrm{E}-03\end{array}$ & $\begin{array}{l}2.4887 \mathrm{E}-03 \\
2.5446 \mathrm{E}-03 \\
2.5468 \mathrm{E}-03 \\
2.5677 \mathrm{E}-03 \\
2.4895 \mathrm{E}-03\end{array}$ & $5.7119 \mathrm{E}-02$ & $\begin{array}{l}5.2846 \mathrm{E}-02 \\
5.5567 \mathrm{E}-02 \\
5.6388 \mathrm{E}-02 \\
5.6385 \mathrm{E}-02 \\
5.0418 \mathrm{E}-02\end{array}$ & $\begin{array}{l}5.6382 \mathrm{E}-02 \\
5.7093 \mathrm{E}-02 \\
5.7121 \mathrm{E}-02 \\
5.7336 \mathrm{E}-02 \\
5.6410 \mathrm{E}-02\end{array}$ \\
\hline 0.3 & 3.182 & $\begin{array}{c}8 \\
16 \\
24 \\
32 \\
40\end{array}$ & $2.0622 \mathrm{E}-03$ & $\begin{array}{l}1.8179 \mathrm{E}-03 \\
1.9218 \mathrm{E}-03 \\
1.9743 \mathrm{E}-03 \\
1.9409 \mathrm{E}-03 \\
1.9805 \mathrm{E}-03\end{array}$ & $\begin{array}{l}1.9961 \mathrm{E}-03 \\
2.0603 \mathrm{E}-03 \\
1.7045 \mathrm{E}-03 \\
1.9068 \mathrm{E}-03 \\
1.8623 \mathrm{E}-03\end{array}$ & $5.1271 \mathrm{E}-02$ & $\begin{array}{l}4.6160 \mathrm{E}-02 \\
4.8765 \mathrm{E}-02 \\
4.9796 \mathrm{E}-02 \\
4.9588 \mathrm{E}-02 \\
5.0190 \mathrm{E}-02\end{array}$ & $\begin{array}{l}5.0341 \mathrm{E}-02 \\
5.1238 \mathrm{E}-02 \\
4.6047 \mathrm{E}-02 \\
4.9122 \mathrm{E}-02 \\
4.8656 \mathrm{E}-02\end{array}$ \\
\hline 0.1 & 5.241 & $\begin{array}{c}8 \\
16 \\
24 \\
32 \\
40\end{array}$ & $1.6177 \mathrm{E}-03$ & $\begin{array}{l}1.4755 \mathrm{E}-03 \\
1.4850 \mathrm{E}-03 \\
1.5005 \mathrm{E}-03 \\
1.5406 \mathrm{E}-03 \\
1.4163 \mathrm{E}-03\end{array}$ & $\begin{array}{l}1.5621 \mathrm{E}-03 \\
9.3918 \mathrm{E}-04 \\
1.4616 \mathrm{E}-03 \\
1.4503 \mathrm{E}-03 \\
1.5091 \mathrm{E}-03\end{array}$ & $4.5065 \mathrm{E}-02$ & $\begin{array}{l}4.0545 \mathrm{E}-02 \\
4.2199 \mathrm{E}-02 \\
4.2898 \mathrm{E}-02 \\
4.4736 \mathrm{E}-02 \\
4.1264 \mathrm{E}-02\end{array}$ & $\begin{array}{l}4.4181 \mathrm{E}-02 \\
3.3944 \mathrm{E}-02 \\
4.2616 \mathrm{E}-02 \\
4.2408 \mathrm{E}-02 \\
4.3276 \mathrm{E}-02\end{array}$ \\
\hline
\end{tabular}

Таблица 3. Результаты расчета сечений $(k=0.5)$ трехслойных подобных сфероидов с диэлектрическими проницаемостями внешней оболочки $\tilde{\varepsilon}_{1}=1.69+0.10 j$, внутренней оболочки $\tilde{\varepsilon}_{2}=2.25+0.15 j$ и ядра $\tilde{\varepsilon}_{3}=2.89+0.20 j$. В колонке „Метод расчета“ в скобках приводится число уравнений $N$, используемых в БСЛАУ при вычислениях

\begin{tabular}{|c|c|c|c|c|c|c|c|}
\hline \multirow{2}{*}{ Случай } & \multirow{2}{*}{$\begin{array}{l}\text { Параметры } \\
\text { сфероида }\end{array}$} & \multicolumn{3}{|c|}{ № оболочки $(i)$} & \multirow{2}{*}{$\begin{array}{c}\text { Метод } \\
\text { расчета }\end{array}$} & \multirow{2}{*}{$\begin{array}{c}\text { Сечение } \\
\text { рассеяния }\end{array}$} & \multirow{2}{*}{$\begin{array}{c}\text { Сечение } \\
\text { поглощения }\end{array}$} \\
\hline & & 1 & 2 & 3 & & & \\
\hline 1 & $\begin{array}{c}a \\
b \\
a / b \\
V[i]\end{array}$ & $\begin{array}{c}1.00 \\
0.80 \\
0.64000\end{array}$ & $\begin{array}{c}0.80 \\
0.64 \\
1.25 \\
0.32768\end{array}$ & $\begin{array}{c}0.60 \\
0.48 \\
0.13824\end{array}$ & $\begin{array}{r}\operatorname{EBCM}(16) \\
\operatorname{EBCM}(24) \\
\operatorname{SVM}(16) \\
\operatorname{SVM}(24)\end{array}$ & $\begin{array}{l}1.68074 \mathrm{E}-02 \\
1.68074 \mathrm{E}-02 \\
1.68074 \mathrm{E}-02 \\
1.68074 \mathrm{E}-02\end{array}$ & $\begin{array}{c}1.05579 \mathrm{E}-01 \\
1.05579 \mathrm{E}-01 \\
1.05579 \mathrm{E}-01 \\
1.05579 \mathrm{E}-01\end{array}$ \\
\hline 2 & $\begin{array}{c}a \\
b \\
a / b \\
V[i] \\
\end{array}$ & $\begin{array}{c}1.00 \\
0.60 \\
0.36000 \\
\end{array}$ & $\begin{array}{c}0.80 \\
0.48 \\
1.67 \\
0.18432 \\
\end{array}$ & $\begin{array}{c}0.60 \\
0.36 \\
0.07776 \\
\end{array}$ & $\begin{array}{r}\operatorname{EBCM}(16) \\
\operatorname{EBCM}(24) \\
\operatorname{SVM}(16) \\
\operatorname{SVM}(24) \\
\end{array}$ & $\begin{array}{l}6.15269 \mathrm{E}-03 \\
2.05085 \mathrm{E}-02 \\
6.01727 \mathrm{E}-03 \\
6.01729 \mathrm{E}-03 \\
\end{array}$ & $\begin{array}{l}6.84322 \mathrm{E}-02 \\
2.89340 \mathrm{E}-01 \\
6.67061 \mathrm{E}-02 \\
6.67063 \mathrm{E}-02 \\
\end{array}$ \\
\hline 3 & $\begin{array}{c}a \\
b \\
a / b \\
V[i]\end{array}$ & $\begin{array}{c}1.00 \\
0.40 \\
0.16000\end{array}$ & $\begin{array}{c}0.80 \\
0.32 \\
2.50 \\
0.08192\end{array}$ & $\begin{array}{c}0.60 \\
0.24 \\
0.03456\end{array}$ & $\begin{array}{r}\operatorname{EBCM}(16) \\
\operatorname{EBCM}(24) \\
\operatorname{SVM}(16) \\
\operatorname{SVM}(24)\end{array}$ & $\begin{array}{l}2.67307 \mathrm{E}-03 \\
5.88321 \mathrm{E}-01 \\
1.37708 \mathrm{E}-03 \\
1.44127 \mathrm{E}-03\end{array}$ & $\begin{array}{r}-1.97017 \mathrm{E}-01 \\
2.82647 \mathrm{E}-02 \\
3.40919 \mathrm{E}-02 \\
3.70293 \mathrm{E}-02\end{array}$ \\
\hline
\end{tabular}

ЕВСМ имеет достаточно высокую точность (три значащие цифры) даже при $N=8$, при этом с увеличением $N$ точность расчетов увеличивается до пяти значащих цифр при $N=40$.
C теоретической точки зрения форма ядра не должна влиять на область применимости ЕBCM и SVM. Тем не менее результаты вычислений показывают, что влияние погрешностей увеличивается с ростом степени 
Таблица 4. То же, что в табл. 3, но для трехслойных подобных псевдосфероидов (ПС)

\begin{tabular}{|c|c|c|c|c|c|c|c|}
\hline \multirow{2}{*}{ Случай } & \multirow{2}{*}{$\begin{array}{c}\text { Параметры } \\
\text { ПС }\end{array}$} & \multicolumn{3}{|c|}{ № оболочки $(i)$} & \multirow{2}{*}{$\begin{array}{c}\text { Метод } \\
\text { расчета }\end{array}$} & \multirow{2}{*}{$\begin{array}{l}\text { Сечение } \\
\text { рассеяния }\end{array}$} & \multirow{2}{*}{$\begin{array}{c}\text { Сечение } \\
\text { поглощения }\end{array}$} \\
\hline & & 1 & 2 & 3 & & & \\
\hline 1 & $\begin{array}{c}a \\
b \\
a / b \\
V[i] \\
\end{array}$ & $\begin{array}{c}.00 \\
0.80 \\
0.83019 \\
\end{array}$ & $\begin{array}{c}0.80 \\
0.64 \\
1.25 \\
0.42506 \\
\end{array}$ & $\begin{array}{c}0.60 \\
0.48 \\
0.17932 \\
\end{array}$ & $\begin{array}{r}\operatorname{EBCM}(16) \\
\operatorname{EBCM}(24) \\
\operatorname{SVM}(16) \\
\operatorname{SVM}(24) \\
\end{array}$ & $\begin{array}{l}.32650 \mathrm{E}-02 \\
2.32650 \mathrm{E}-02 \\
2.32650 \mathrm{E}-02 \\
2.32650 \mathrm{E}-02 \\
\end{array}$ & $\begin{array}{l}1.14084 \mathrm{E}-01 \\
1.14084 \mathrm{E}-01 \\
1.14084 \mathrm{E}-01 \\
1.14084 \mathrm{E}-01\end{array}$ \\
\hline 2 & $\begin{array}{c}a \\
b \\
a / b \\
V[i] \\
\end{array}$ & $\begin{array}{c}1.00 \\
0.60 \\
0.71367 \\
\end{array}$ & $\begin{array}{c}0.80 \\
0.48 \\
1.67 \\
0.36540 \\
\end{array}$ & $\begin{array}{c}0.60 \\
0.36 \\
0.15415 \\
\end{array}$ & $\begin{array}{r}\operatorname{EBCM}(16) \\
\operatorname{EBCM}(24) \\
\operatorname{SVM}(16) \\
\operatorname{SVM}(24) \\
\end{array}$ & $\begin{array}{l}1.58393 \mathrm{E}-02 \\
1.58315 \mathrm{E}-02 \\
1.58387 \mathrm{E}-02 \\
1.58387 \mathrm{E}-02 \\
\end{array}$ & $\begin{array}{l}9.10351 \mathrm{E}-02 \\
9.11799 \mathrm{E}-02 \\
9.10235 \mathrm{E}-02 \\
9.10235 \mathrm{E}-02 \\
\end{array}$ \\
\hline 3 & $\begin{array}{c}a \\
b \\
a / b \\
V[i]\end{array}$ & $\begin{array}{c}1.00 \\
0.40 \\
0.64033\end{array}$ & $\begin{array}{c}0.80 \\
0.32 \\
2.50 \\
0.32785\end{array}$ & $\begin{array}{c}0.60 \\
0.24 \\
0.13831\end{array}$ & $\begin{array}{r}\operatorname{EBCM}(16) \\
\operatorname{EBCM}(24) \\
\operatorname{SVM}(16) \\
\operatorname{SVM}(24)\end{array}$ & $\begin{array}{l}2.78183 \mathrm{E}+00 \\
5.86489 \mathrm{E}+01 \\
1.20927 \mathrm{E}-02 \\
1.20924 \mathrm{E}-02\end{array}$ & $\begin{array}{r}-5.51304 \mathrm{E}-01 \\
1.44310 \mathrm{E}+00 \\
7.80336 \mathrm{E}-02 \\
7.80327 \mathrm{E}-02\end{array}$ \\
\hline
\end{tabular}

Таблица 5. То же, что в табл. 3, но для трехслойных подобных улиток Паскаля (УП)

\begin{tabular}{|c|c|c|c|c|c|c|c|}
\hline \multirow{2}{*}{ Случай } & \multirow{2}{*}{$\begin{array}{c}\text { Параметры } \\
\text { УП }\end{array}$} & \multicolumn{3}{|c|}{ № оболочки $(i)$} & \multirow{2}{*}{$\begin{array}{c}\text { Метод } \\
\text { расчета }\end{array}$} & \multirow{2}{*}{$\begin{array}{c}\text { Сечение } \\
\text { рассеяния }\end{array}$} & \multirow{2}{*}{$\begin{array}{c}\text { Сечение } \\
\text { поглощения }\end{array}$} \\
\hline & & 1 & 2 & 3 & & & \\
\hline \multirow{2}{*}{1} & $\begin{array}{l}a \\
\epsilon \\
\end{array}$ & 1.00 & $\begin{array}{c}0.80 \\
0.1 \\
\end{array}$ & 0.60 & \multirow{2}{*}{$\begin{array}{r}\operatorname{EBCM}(16) \\
\operatorname{EBCM}(24) \\
\operatorname{SVM}(16) \\
\operatorname{SVM}(24) \\
\end{array}$} & \multirow{2}{*}{$\begin{array}{l}3.77283 \mathrm{E}-02 \\
3.77283 \mathrm{E}-02 \\
3.77283 \mathrm{E}-02 \\
3.77283 \mathrm{E}-02 \\
\end{array}$} & \multirow{2}{*}{$\begin{array}{l}1.51145 \mathrm{E}-01 \\
1.51145 \mathrm{E}-01 \\
1.51145 \mathrm{E}-01 \\
1.51145 \mathrm{E}-01 \\
\end{array}$} \\
\hline & $V[i]$ & 1.01000 & 0.51712 & 0.21816 & & & \\
\hline \multirow{2}{*}{2} & $\begin{array}{l}a \\
\epsilon \\
\end{array}$ & 1.00 & $\begin{array}{c}0.80 \\
0.3 \\
\end{array}$ & 0.60 & \multirow{2}{*}{$\begin{array}{r}\operatorname{EBCM}(16) \\
\operatorname{EBCM}(24) \\
\operatorname{SVM}(16) \\
\operatorname{SVM}(24) \\
\end{array}$} & \multirow{2}{*}{$\begin{array}{l}4.32647 \mathrm{E}-02 \\
4.32647 \mathrm{E}-02 \\
4.32647 \mathrm{E}-02 \\
4.32647 \mathrm{E}-02 \\
\end{array}$} & \multirow{2}{*}{$\begin{array}{l}1.60788 \mathrm{E}-01 \\
1.60788 \mathrm{E}-01 \\
1.60788 \mathrm{E}-01 \\
1.60788 \mathrm{E}-01 \\
\end{array}$} \\
\hline & $V[i]$ & 1.09000 & 0.55808 & 0.23544 & & & \\
\hline \multirow{2}{*}{3} & $\begin{array}{l}a \\
\epsilon \\
\end{array}$ & 1.00 & $\begin{array}{c}0.80 \\
0.5 \\
\end{array}$ & 0.60 & \multirow{2}{*}{$\begin{array}{r}\operatorname{EBCM}(16) \\
\operatorname{EBCM}(24) \\
\operatorname{SVM}(16) \\
\operatorname{SVM}(24) \\
\end{array}$} & \multirow{2}{*}{$\begin{array}{l}5.59690 \mathrm{E}-02 \\
5.58229 \mathrm{E}-02 \\
5.57912 \mathrm{E}-02 \\
5.57912 \mathrm{E}-02 \\
\end{array}$} & \multirow{2}{*}{$\begin{array}{l}1.81033 \mathrm{E}-01 \\
1.81940 \mathrm{E}-01 \\
1.81158 \mathrm{E}-01 \\
1.81158 \mathrm{E}-01 \\
\end{array}$} \\
\hline & $V[i]$ & 1.25000 & 0.64000 & 0.27000 & & & \\
\hline \multirow{2}{*}{4} & $\begin{array}{l}a \\
\epsilon\end{array}$ & 1.00 & $\begin{array}{c}0.80 \\
0.7\end{array}$ & 0.60 & \multirow{2}{*}{$\begin{array}{r}\operatorname{EBCM}(16) \\
\operatorname{EBCM}(24) \\
\operatorname{SVM}(16) \\
\operatorname{SVM}(24)\end{array}$} & \multirow{2}{*}{$\begin{array}{l}8.28309 \mathrm{E}+07 \\
5.56686 \mathrm{E}+10 \\
7.82135 \mathrm{E}-02 \\
5.28929 \mathrm{E}+01\end{array}$} & \multirow{2}{*}{$\begin{array}{r}1.14311 \mathrm{E}+04 \\
4.41757 \mathrm{E}+05 \\
2.13418 \mathrm{E}-01 \\
-3.55032 \mathrm{E}+01\end{array}$} \\
\hline & $V[i]$ & 1.49000 & 0.76288 & 0.32184 & & & \\
\hline
\end{tabular}

асферичности ядра. Напомним, что сверхточные расчеты для двухслойных конфокальных сфероидов [18] с учетом 300 значащих цифр при $N=400$ свидетельствуют о потере 110 цифр при $a_{1} / b_{1}=1.4,220$ цифр при $a_{1} / b_{1}=1.8,276$ цифр при $a_{1} / b_{1}=2.3$ и отсутствии даже 1 верной цифры при $a_{1} / b_{1}=\sqrt{2}+1$. Наши расчеты показывают, что ближе к границе области применимости при отношении полуосей частицы $a_{1} / b_{1}=2$ влияние вычислительных погрешностей становится еще более заметно по сравнению со случаем $a_{1} / b_{1}=1.43$, причем ошибки увеличиваются как с ростом $N$, так и с увеличением отношения полуосей ядра $a_{2} / b_{2}$ (табл. 2). Здесь размерность симметричного блока для ЕВСМ уменьшается от $N_{T}=9$ для первого случая, $N_{T}=5$ для второго до $N_{T}=3$ для третьего и четвертого. В последнем случае лишь при $N=32$ симметричный блок для ЕВСМ имеет размерность $N_{T}=3$ с погрешностью чуть более $10 \%$. Сравнение результатов для ЕВСМ и SVM показывает, что SVM дает более точные результаты в более широкой области изменения параметров, при этом $N_{T}(\mathrm{SVM})>N_{T}(\mathrm{EBCM})$. Для рассматриваемых частиц максимальные значения размерности симметричного блока для SVM уменьшаются от $N_{T}=15(N=32)$ для первого случая, $N_{T}=13(N=32)$ для второго, $N_{T}=9(N=24)$ для третьего до $N_{T}=5(N=16)$ для четвертого. В последнем случае симметричный блок для SVM едва заметен: $N_{T}=3$ при $N=8$ с последующим исчезновением.

Для подобных трехслойных сфероидов $\left(a_{1} / b_{1}=a_{2} / b_{2}=\right.$ $\left.=a_{3} / b_{3}\right)$ результаты численных расчетов представлены в табл. 3. Заметим, что данные сфероиды являются неконфокальными, при этом область применимости 
определяется условием (20). Анализ данных показывает, что снова SVM дает более точные результаты. Во втором случае для ЕВСМ лишь при $N=16$ размерность $N_{T}=3$, в то время как размерность симметричного блока для SVM $N_{T}=9$ при $N=16$ и $N_{T}=15$ при $N=24$. Более того, даже вне области применимости $\left(a_{1} / b_{1}=2.5\right)$ SVM дает сравнительно пригодные результаты: $N_{T}=3$ при $N=16$. Здесь указанный метод следует рассматривать как приближенный, точность результатов которого следует дополнительно проверять.

Рассмотрим осесимметричные частицы, форма которых может быть невыпуклой. Уравнение поверхности сплюснутого псевдосфероида с полуосями $a=1$ и $b \leq 1$ можно записать в виде

$$
r(\theta)=a \sqrt{1-\epsilon^{2} \cos ^{2} \theta}, \quad \epsilon^{2}=1-b^{2} / a^{2} \geq 0 .
$$

Данные частицы получаются в результате инверсии относительно начала координат вытянутых сфероидов с теми же полуосями и фокусным расстоянием $d=2 \sqrt{a^{2}-b^{2}}$. Объем частицы можно вычислить по формуле

$$
V=\frac{2 \pi}{3} a^{3} \int_{-1}^{1}\left(1-\epsilon^{2} x^{2}\right)^{3 / 2} d x .
$$

Псевдосфероиды имеют выпуклую форму при $a / b<\sqrt{2} \approx 1.41, \quad$ т.е. $\epsilon^{2}<1 / 2=0.5, \quad$ а условие применимости ЕВСМ для однородных частиц записывается следующим образом: $a / b<\sqrt{2}+1 \approx 2.41$, т.е. $\epsilon^{2}<2(\sqrt{2}-1) \approx 0.83 \quad[20]$. Для трехслойных подобных псевдосфероидов $\left(a_{1} / b_{1}=a_{2} / b_{2}=a_{3} / b_{3}\right)$ имеем

$$
\begin{gathered}
\sigma_{1}^{(1)}<\sigma_{2}^{(3)}, \\
\sigma_{1}^{(1)}=\frac{d_{1}}{4}, \\
\sigma_{2}^{(3)}=\frac{2 a_{3} b_{3}}{d_{3}} .
\end{gathered}
$$

Решая эту систему уравнений с учетом соотношения подобия $a_{3} / a_{1}=b_{3} / b_{1}=d_{3} / d_{1}=k_{3}$, получим условие применимости сферического базиса:

$$
\frac{a_{j}}{b_{j}}<k_{3}+\sqrt{k_{3}^{2}+1}
$$

Обращаясь к табл. 4, для всех случаев получим $k_{3}=0.6, a_{j} / b_{j}<1.766$, при этом первые два удовлетворяют данному условию. Заметим, что во втором случае слои являются невыпуклыми. Третий случай выпадает из области применимости, и для него предлагаемый подход следует считать неким приближением. Здесь метод ЕВCM явно не работает, в то время как SVM дает хорошие результаты, а именно тест на размерность симметричного блока дает $N_{T}=13$ при $N=16$ и $N_{T}=7$ при $N=24$. Заметим, что при рассмотрении трехслойных конфокальных псевдосфероидов $\left(d_{1}=d_{2}=d_{3}\right)$ условие применимости (28) накладывается на ядро частицы, т. е. для псевдосфероидов с одинаковыми ядрами случай с подобными слоями представляется более сложным для использования сферического базиса с целью расчета светорассеяния.

В заключении рассмотрим чебышевские частицы с одним максимумом (улитки Паскаля), для которых уравнение поверхности записывается следующим образом:

$$
r(\theta)=a(1+\epsilon \cos \theta),
$$

где $a-$ радиус невозмущенного шара, при этом параметр возмущения $|\epsilon|<1$. Объем улитки Паскаля вычисляется по формуле

$$
V=\frac{4 \pi}{3} a^{3}\left(1+\epsilon^{2}\right)
$$

Данные частицы получаются в результате инверсии вытянутых сфероидов относительно одного из фокусов. Они являются выпуклыми при $\epsilon<1 / 2=0.5$, а условие применимости ЕВСМ для однородных улиток имеет вид $\epsilon<1 / \sqrt{2} \approx 0.707$ [21]. Для трехслойных подобных улиток $\left(\epsilon_{1}=\epsilon_{2}=\epsilon_{3}\right)$ условие (18) с учетом особенностей поверхностей можно записать в виде

$$
\begin{gathered}
\sigma_{1}^{(1)}<\sigma_{2}^{(3)}, \\
\sigma_{1}^{(1)}=a_{1} \frac{\left|\epsilon_{j}\right|}{2}, \\
\sigma_{2}^{(3)}=a_{3} \frac{1-\epsilon_{j}^{2}}{2\left|\epsilon_{j}\right|} .
\end{gathered}
$$

Решение этой системы, т. е. условие применимости сферического базиса, имеет вид

$$
\left|\epsilon_{j}\right|<\sqrt{\frac{1}{4 k_{3}^{2}}+1}-\frac{1}{2 k_{3}},
$$

где для рассматриваемых слоистых частиц коэффициент подобия $k_{3}=a_{3} / a_{1}=0.6$ и соотношение (31) сводится к неравенству $\epsilon<0.468$. Первые два случая, представленные в табл. 5, удовлетворяют этому требованию и дают отличные результаты. Третий случай немного выходит за пределы области применимости, поэтому полученные результаты следует рассматривать как приближенное решение. Метод SVM снова предпочтительнее, при этом тест на размерность симметричного блока дает $N_{T}=11$ при $N=16$ и $N_{T}=5$ при $N=24$. В последнем случае результат SVM при $N=16$ заслуживает внимания, так как симметричный блок имеет размерность $N_{T}=4$, причем все элементы не равны нулю в отличие от ранее рассмотренных частиц.

\section{Заключение}

На основании аналитического и численного анализа построенного приближения Релея с использованием SVM-решения электростатической задачи для многослойных осесимметричных частиц можно сделать вывод 
о преимуществе данной схемы по сравнению со схемой, в которой применяется ЕВСМ-решение. Данный алгоритм расчета сечений рассеяния и поглощения применим в более широкой области изменения параметров рассматриваемых частиц.

Работа была поддержена в 2017 г. грантом ГУАП и грантом РФФИ 16-02-00194а.

\section{Список литературы}

[1] Борен К., Хабфмен Д. Поглощение и рассеяние света малыми частицами. М.: Мир, 1986.

[2] Морс Ф.М., Фешбах Г. Методы теоретической физики. М.: ИЛ, 1958.

[3] Ландау Л.Д., Лившии, Е.М. Электродинамика сплошных сред. М.: Наука, 1982.

[4] Фарафонов В.Г. // Опт. и спектр. 2000. Т. 88. № 3. С. 441.

[5] Фарабонов В.Г. // Опт. и спектр. 2001. Т. 90. № 4. С. 646.

[6] Posselt B., Farafonov V.G., Il'in V.B., Prokopjeva M.S. // Meas. Sci. Technol. 2002. V. 13. P. 256.

[7] Фарафонов В.Г., Устимов В.И., Соколовская М.В. // Опт. и спектр. 2016. Т. 120. № 3. С. 470.

[8] Farafonov V., Ilin V., Ustimov V., Volkov E. // Adv. Math. Phys. 2017. V. 2017. Article ID 7862462. P. 1.

[9] Фарафонов В.Г., Устимов В.И. // Опт. и спектр. 2016. T. 122. № 2. C. 287.

[10] Фарафонов В.Г., Винокуров А.А., Ильин В.Б. // Опт. и спектр. 2007. Т. 102. № 6. С. 1006.

[11] Farafonov V.G., Il'in V., Ustimov V., Prokopjeva M. // J. Quant. Spectr. Rad. Trasf. 2016. V. 178. P. 176.

[12] Фарафонов В.Г., Винокуров А.А. // Опт. и спектр. 2008. T. 105. № 2. C. 318.

[13] Vinokurov A.A., Farafonov V.G., Il'in V.B. // J. Quant. Spectr. Rad. Transf. 2009. V. 110. P. 1356.

[14] Фарафонов В.Г. // Опт. и спектр. 2014. Т. 117. № 6. С. 949.

[15] Фарафонов В.Г., Устимов В.И. // Опт. и спектр. 2015. T. 119. № 6. C. 1020.

[16] Farafonov V.G., Ilin V.B. // J. Quant. Spectr. Rad. Trast. 2014. V. 146. P. 244.

[17] Кюркчан А.Г., Смирнова Н.И. Математическое моделирование в теории дифракции с использованием априорной информации об аналитических свойствах решения. М.: ИД Медиа Паблишер, 2014.

[18] Фарафбонов В.Г., Ильин В.Б. // Опт. и спектр. 2013. Т. 115. № 5. C. 836.

[19] Farafonov V.G., Sokolovskaya M.V. // J. Math. Sci. 2013. V. 194. P. 104.

[20] Фарафонов В.Г., Устимов В.И. // Опт. и спектр. 2015. T. 119. № 5. C. 834.

[21] Фарафонов В.Г., Устимов В.И. // Опт. и спектр. 2015. T. 118. № 3. C. 469. 